\title{
Basic Fibroblast Growth Factor Augments Podocyte Injury and Induces Glomerulosclerosis in Rats with Experimental Membranous Nephropathy
}

\author{
Jürgen Floege, Wilhelm Kriz, * Matthias Schulze, Martin Susani, ${ }^{*}$ Dontscho Kerjaschki, ${ }^{*}$ Andrew Mooney," \\ William G. Couser, ${ }^{\S}$ and Karl M. Koch \\ Division of Nephrology, Medizinische Hochschule, 30623 Hannover, Germany; *Department of Anatomy, University of Heidelberg, \\ 69120, Heidelberg, Germany; ${ }^{\ddagger}$ Department of Pathology, University of Vienna, 1090 Vienna, Austria; ${ }^{\S}$ Division of Nephrology, \\ University of Washington, Seattle, Washington 98105; and "Division of Nephrology, University Hospital, Nottingham NG7 2UH, \\ United Kingdom
}

\begin{abstract}
Podocyte injury is believed to contribute to glomerulosclerosis in membranous nephropathy. To identify the factors involved, we investigated the effects of basic fibroblast growth factor (bFGF), a cytokine produced by podocytes, on rats with membranous nephropathy (passive Heymann nephritis [PHN]). All rats received a daily i.v. bolus of $10 \mu \mathrm{g}$ bFGF or vehicle from days 3-8 after PHN induction. In proteinuric PHN rats on day 8, bFGF injections further increased proteinuria. Podocytes of bFGF-injected PHN rats showed dramatic increases in mitoses, pseudocyst formation, foot process retraction, focal detachment from the glomerular basement membrane, and desmin expression. bFGF injections in PHN rats did not alter antibody or complement deposition or glomerular leukocyte influx. bFGF-injected PHN rats developed increased glomerulosclerosis when compared with control PHN rats. Also, bFGF induced proteinuria and podocyte damage in rats injected with $10 \%$ of the regular PHN-serum dose. None of these changes occurred in bFGF-injected normal rats, complement-depleted PHN rats or rats injected with 5\% of the regular PHNserum dose. These divergent bFGF effects were explained in part by upregulated glomerular bFGF receptor expression, induced by PHN serum. Thus, bFGF can augment podocyte damage, resulting in increased glomerular protein permeability and accelerated glomerulosclerosis. This bFGF action is confined to previously injured podocytes. Release of bFGF from glomerular sources (including podocytes themselves) during injury may represent an important mechanism by which podocyte damage is enhanced or becomes self sustained. (J. Clin. Invest. 1995. 96:2809-2819.) Key words: glomerulonephritis - FGF receptor • HIV-nephropathy $\bullet$ growth factor $\bullet$ epithelial cells
\end{abstract}

Parts of this study have been presented in abstract form at the 27th Annual Meeting of the American Society of Nephrology, Orlando, FL, 26-29 October 1994.

Address correspondence to Jürgen Floege, Division of Nephrology 6840, Medizinische Hochschule, 30623 Hannover, Germany. Phone: 511-5323656; FAX: 511-552366.

Received for publication 22 November 1994 and accepted in revised form 22 August 1995.

J. Clin. Invest.

(C) The American Society for Clinical Investigation, Inc.

$0021-9738 / 95 / 12 / 2809 / 11 \$ 2.00$

Volume 96, December 1995, 2809-2819

\section{Introduction}

Membranous nephropathy is one of the most common types of glomerulonephritis in humans (1). Studies on Heymann nephritis, a rat model of membranous nephropathy, as well as subsequent clinical studies have established that the acute glomerular damage in membranous nephropathy results from the formation of subepithelial immune complexes, complement activation, and finally, damage to the visceral glomerular epithelial cells (podocytes) (reviewed in references 1 and 2). In contrast to the pathogenesis of the acute phase of the disease, relatively little is known about the mechanism(s) underlying the development of progressive glomerulosclerosis and renal insufficiency, occurring in $\sim 50 \%$ of the patients with membranous nephropathy (1).

Recently, we have become interested in the role of basic fibroblast growth factor (bFGF, ${ }^{1}$ also termed FGF2) during glomerular disease $(3,4)$. Basic FGF is a pleiotropic cytokine, which is constitutively expressed in many cell types (5), including glomerular mesangial cells (3). Upon ( sub-)lethal cell injury, basic FGF is released (reviewed in reference 6 ) and we have suggested that this may be an important step in the initiation of mesangioproliferative glomerulonephritis (3). Interestingly, in those studies, prior injury to the mesangial cells was required to render them susceptible to the mitogenic action of bFGF (3).

In addition to glomerular mesangial cells, immunoreactivity for bFGF can also be detected in podocytes as well as in parietal glomerular epithelial cells (7). Similar to its action on mesangial cells in vitro (8), bFGF was also found to act as a mitogen on glomerular epithelial cells in culture (7). In the present study we have therefore examined the questions of whether bFGF may act as a mitogen on podocytes in vivo and whether the bFGF-induced proliferation might lead to an aggravation of preexisting glomerular injury resulting in permanent damage. For these studies, experimental membranous nephropathy was induced in rats by injecting an antiserum to a crude tubular extract, termed Fx1A (passive Heymann nephritis [PHN]) (9). Our data show that under these circumstances bFGF induced mitoses and pseudocyst formation in podocytes, but not in parietal glomerular epithelial cells. This action of bFGF was restricted to rats with prior complement-mediated podocyte injury and may, in part, result from upregulated bFGF receptor expression in injured podocytes. The aggravation of morphological

1. Abbreviations used in this paper: bFGF, basic fibroblast growth factor; CVF, cobra venom factor; GBM, glomerular basement membrane; PAS, periodic acid Schiff; PHN, passive Heymann nephritis. 


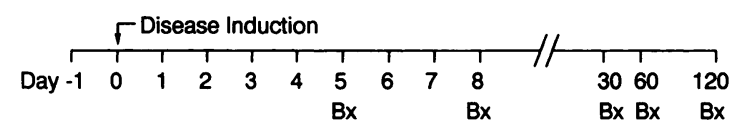

\begin{tabular}{|c|c|c|c|c|}
\hline A & $\underset{1 \mathrm{ml} \text { Anti-Fx1A }}{\Lambda}$ & $\bigcup_{\text {bFGF or Vehicle }} \underset{\Lambda}{\Lambda}$ & Sacrifice 1 & 蛋 \\
\hline$[\mathrm{B}$ & $\underbrace{}_{\text {CVF }} \operatorname{lm}_{\text {Anti-FxiA }}$ & $\begin{array}{l}\text { bFGF or Lsacrifice } \\
\text { Vehicle }\end{array}$ & & \multirow{3}{*}{ 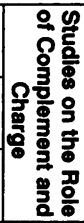 } \\
\hline c & $\underset{1 \mathrm{ml} \text { Anti-gp330 }}{\Lambda}$ & $\Delta \|_{\text {bFGF }}^{\Lambda} \Lambda \Delta$ & $L_{\text {Sacrifice }}$ & \\
\hline D & $\bigsqcup_{1 \mathrm{ml} \text { Anti-Fx1A }}^{\Lambda}$ & $\Delta \underset{\text { Poly-D-Lysine }}{\Lambda} \underset{\Delta}{\Lambda} \Lambda$ & Sacrifice -1 & \\
\hline
\end{tabular}

\begin{tabular}{|c|c|c|c|c|}
\hline $\mathbf{E}$ & $\Delta_{0.1 \mathrm{ml} \text { Anti-Fx1A }}^{\Delta}$ & $\Lambda_{\text {bFGF or Vehicle }} \|_{\Delta}$ & Sacrifice -1 & 옿욜을 \\
\hline $\mathbf{F}$ & $\bigsqcup_{0.05 \mathrm{ml} \text { Anti-Fx1A }}^{\Lambda}$ & $\boldsymbol{\Delta}_{\text {bFGF or Vehicle }} \boldsymbol{\Lambda}$ & Sacrifice $\rfloor$ & 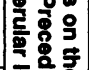 \\
\hline $\mathrm{G}$ & $\stackrel{1}{\text { PBS }}$ & $\Delta \backslash \underset{b F G F}{\Lambda} \Delta \Lambda$ & Sacrifice & \\
\hline
\end{tabular}

Figure 1. Experimental design. Outline of the experimental design showing the various groups $(A-G)$ of rats studied. Bx, renal biopsy. Arrowheads denote intravenous bolus injections.

injury was associated with increased proteinuria and an accelerated development of glomerulosclerosis.

\section{Methods}

Experimental design. Male Sprague Dawley rats (Zentralinstitut für Versuchstierkunde, Hannover, Germany), weighing $\sim 200$ grams at the start of the experiment, were used for all experiments. Animal experiments, and the experimental design were approved by the local review boards.

Unless stated otherwise, the following protocol was used in all experimental groups (Fig. 1): PHN was induced using a sheep antibody to Fx1A as described (10). Anti-Fx1A or control antibody or PBS was injected intravenously at day 0 . From day 3 to 7 after disease induction, rats received daily i.v. bolus injections of either a 500- $\mu$ l vehicle ( 10 $\mathrm{mM}$ sodium citrate buffer, $\mathrm{pH} 6.0$; diluted 1:500 in PBS $\mathrm{pH}$ 7.4) or a 500- $\mu \mathrm{l}$ vehicle containing $10 \mu \mathrm{g}$ human recombinant bFGF (kindly provided by Synergen, Boulder, CO). Surgical (maximum two per rat) or autoptic renal cortical biopsies as well as serum samples were obtained on days 5, 8, 30, 60, and 120 after disease induction. 24-h urine collections were performed on days $3,8,13,30,60$, and 120 after disease induction. To allow the measurement of urinary C5b-9 concentration, urine was collected in $10 \%(\mathrm{vol} / \mathrm{vol})$ of a $0.2 \mathrm{M}$ Tris-buffer containing a mixture of protease inhibitors (benzamidine, $\epsilon$-aminocaproic acid, EDTA, aprotinin) as previously described (11).

The following experimental groups, depicted schematically in Fig. 1, were studied: (a) 24 rats received the full dose, i.e., $1.0 \mathrm{ml}$, of antiFx1A (subsequently referred to as "PHN rats"). (b) Eight PHN rats prior to disease induction were depleted of complement with cobra venom factor (CVF) (Naja naja kaounthia; Diamedix Corp., Miami, FL) as previously described (12). Rats then received anti-Fx1A followed by two bolus injections of bFGF or vehicle (days 3 and 4) before killing on day 5. (c) Four rats received an i.v. injection of anti-gp 330 IgG at a dose which was equivalent to the amount of anti-gp 330 contained in $1 \mathrm{ml}$ of anti-Fx1A antiserum (13). gp 330 is a podocyte antigen recognized by anti-Fx1A antibody (2). However, although antigp $330 \mathrm{IgG}$ binds to podocytes and induces the formation of typical subepithelial immune complexes, it does not result in complement activation or proteinuria (2). Anti-gp $330 \mathrm{IgG}$ for this study was isolated from the sheep anti-Fx1A antiserum by affinity chromatography as pre- viously described (13). Anti-gp 330-injected rats then received five bolus injections of bFGF as described above. Renal biopsies were obtained at day 5 and at death (day 8). (d) Four rats received $1 \mathrm{ml}$ anti-Fx1A, followed by five bolus injections of $10 \mu \mathrm{g}$ poly-D-lysine (hydrobromide, mol wt 15,000-30,000, pI $\sim 10.5$; Sigma Chemical Co., Deisenhofen, Germany). Renal biopsies were obtained at days 5 , 8 , and at time of death (day 120 ). (e) 24 rats received $10 \%$ of the full dose of anti-Fx1A antiserum, i.e., $0.1 \mathrm{ml}$ per rat, before the bFGF or vehicle injections. Pilot experiments had demonstrated that at this dose of anti-Fx1A antiserum no proteinuria developed during the heterologous phase of the disease (through day 6), while proteinuria did develop during the autologous phase after day $6 .(f) 12$ rats received $5 \%$ of the regular amount of anti-Fx1A antiserum, i.e., $0.05 \mathrm{ml}$ per rat before the bFGF or vehicle injections. In pilot experiments this dose did not induce proteinuria during either the heterologous or the autologous phase. $(g)$ Four rats received an injection of $1 \mathrm{ml}$ PBS at the start of the experiment, followed by five bFGF bolus injections as described above. Renal biopsies were obtained on days 5,8 , and 120 .

Renal morphology. Tissue for light microscopy and immunoperoxidase staining was fixed in methyl Carnoy's solution (14) and embedded in paraffin. $4-\mu \mathrm{m}$ sections were stained with the periodic acid Schiff (PAS) reagent and counterstained with hematoxylin. For each biopsy over 30 cross sections (range 30-100) of consecutive cortical glomeruli containing $>20$ discrete capillary segments each were evaluated by one of the authors, who was unaware of the origin of the slides. The number of glomerular mitoses was counted separately in 3 locations: those located within the core of the glomerular tuft, those located at the outer edge of the tuft and those within parietal glomerular epithelial cells. Mean values per biopsy were then calculated and extrapolated to 100 glomeruli. To obtain mean numbers of mitoses in cells of the cortical tubulointerstitium, over 30 grid fields (range 30-50), measuring 0.1 $\mathrm{mm}^{2}$ each, were evaluated (grid parts containing glomerular cross sections were ignored in this evaluation). Glomerular apoptoses were recognized as described previously (15) as cells with densely staining nuclei, smaller than any others, with no nuclear heterogeneity on racking up and down on the focussing ring of the microscope. These cells were sometimes in a "halo" of tissue from which they had retracted. Glomerular matrix expansion was assessed by staining sections with silver methenamine. The percentage of glomeruli exhibiting focal or global glomerulosclerosis was determined as previously described (16).

Immunoperoxidase staining. 4- $\mu \mathrm{m}$ sections of methyl Carnoy's fixed biopsy tissue were processed by a direct or indirect immunoperoxidase technique as previously described (14). Primary antibodies included: (a) ED1 (Camon, Wiesbaden, Germany), a murine monoclonal IgG antibody to a cytoplasmic antigen present in monocytes, macrophages and dendritic cells (17); (b) RP-3 (kind gift of F. Sendo, Yamagata, Japan ), a murine monoclonal IgM antibody to rat neutrophils (18); (c) OX-22 (Serotec Ltd., Kidlington, United Kingdom), a murine monoclonal IgG antibody to the high molecular weight form of the rat common leukocyte antigen expressed on $B$ lymphocytes and most $T$ lymphocytes; $(d)$ DE6, a murine monoclonal $\operatorname{IgG}_{1}$ antibody against recombinant human bFGF (kindly provided by T. Reilly; DuPont-Merck, Wilmington, DE) (19), which cross-reacts with rat bFGF (3); (e) D33, a murine monoclonal $\mathrm{IgG}_{1}$ antibody against human muscle desmin (Dako Corp., Glostrup, Denmark); $(f) 1 \mathrm{~A} 4$, a murine monoclonal $\operatorname{IgG}_{2 \mathrm{a}}$ antibody to an $\mathrm{NH}_{2}$-terminal synthetic decapeptide of $\alpha$-smooth muscle actin (Dako Corp.); $(g)$ an IgG fraction of polyclonal rabbit anti-rat laminin (Chemicon, Temecula, CA); $(h)$ affinity purified polyclonal goat anti-human/ bovine type IV collagen (Southern Biotechnology, Birmingham, AL).

For all biopsies, negative controls consisted of substitution of the primary antibody with equivalent concentrations of an irrelevant murine monoclonal antibody or normal rabbit or goat IgG.

Mean values per biopsy were calculated for the number of leukocytes per glomerular cross section (see comment above). To obtain mean numbers of infiltrating leukocytes in the cortical tubulointerstitium, 30 grid fields, measuring $0.1 \mathrm{~mm}^{2}$ each, were evaluated. For the evaluation of the immunoperoxidase stains for desmin, $\alpha$-smooth muscle actin, bFGF, laminin and type IV collagen, each glomerular tuft was graded 
semiquantitatively as described previously (20), and the mean score per biopsy was calculated. Each score reflects mainly changes in the extent rather than intensity of glomerular staining: 0 , Diffuse, very weak or absent glomerular staining. No localized increases of staining; $1+$, as " 0 ", but up to $25 \%$ of the glomerular tuft showing focally increased staining; $2+, 25-50 \%$ of the glomerular tuft showing focally increased staining; $3+, 50-75 \%$ of the glomerular tuft showing focally increased staining; $4+,>75 \%$ of the glomerular tuft showing focally increased staining.

To assess podocytes separately, the glomerular immunostains for desmin and bFGF were also graded according to the extent of cellular staining at the outer edge of the glomerular tuft: 0 , Very weak or absent staining; $1+$, Strong staining of up to $25 \%$ of the cells lining the glomerular tuft; $2+, 25-50 \%$ of the cells lining the glomerular tuft demonstrating strong staining; $3+, 50-75 \%$ of the cells lining the glomerular tuft demonstrating strong staining; $4+,>75 \%$ of the cells lining the glomerular tuft demonstrating strong staining.

Immunofluorescence staining. Immunofluorescence detection of glomerular sheep IgG or complement $\mathrm{C} 3$ was carried out on 4- $\mu \mathrm{m}$ sections of frozen kidney tissue using an indirect immunofluorescence procedure (21). Primary antibodies included polyclonal donkey anti-sheep IgG (Amersham Corp., Braunschweig, Germany) and polyclonal rabbit anti-human C3c (Dako Corp.).

For the immunofluorescent detection of rat bFGF receptor a polyclonal rabbit antibody to an external region peptide of the $f g$ receptor (Upstate Biotechnology Inc., Lake Placid, NY) was used on $0.5-\mu \mathrm{m}$ frozen sections. This antibody has been previously shown to recognize rat bFGF receptor (22). Bound primary antibody was detected with affinity-purified, biotinylated goat anti-rabbit IgG (Vector Laboratories, Inc., Burlingame, CA) and streptavidin-FITC (Amersham Corp.). In control sections, the bFGF receptor antibody was replaced by normal rabbit serum.

For the evaluation of immunofluorescent stains, glomerular cross sections were graded semiquantitatively as either $\mathbf{0}$ (indistinguishable from control) or positive according to the degree of glomerular fluorescence $(1+$, weak; $2+$, moderate; $3+$, intense).

Quantification of glomerular $\lg G$ deposition. To quantify glomerular deposition of sheep (anti-Fx1A) IgG, 4- $\mu$ m sections of frozen kidney were stained for sheep IgG as stated above. Sections were then scanned by confocal microscopy using an ACAS Ultima Interactive Laser Cytometer (Meridian Instruments, Inc., Okemos, MI), which permits quantitative analyses of fluorescence intensity. An image field of $3.2 \mathrm{~mm}^{2}$ was scanned for each section; where $<15$ glomeruli were present two fields were scanned. Using the Meridian image analysis system, the fluorescence intensity over individual glomeruli was then analyzed. Data from a minimum of 13 individual glomeruli per animal were averaged. Results are expressed as average fluorescence units emitted per glomerulus.

Electron microscopy. Tissue was cut into pieces of $\sim 1 \mathrm{~mm}^{3}$ and fixed for at least $24 \mathrm{~h}$ in $1.25 \%$ glutaradehyde (Merck, Darmstadt, Germany), dissolved in a buffer containing $0.04 \mathrm{M}$ cacodylic acid (sodium salt; Serva, Heidelberg, Germany), pH 7.2. Tissue was then processed for transmission electron microscopy by standard procedures.

Immunoelectron microscopy. Immunoelectron microscopy with the polyclonal rabbit antibody to an external region peptide of the $f l g$ receptor (Upstate Biotechnology Inc.) was performed on frozen tissue as described previously (23).

Measurement of urinary C5b-9. Detection of C5b-9 in urine was performed by an ELISA that utilizes antibodies to a neoantigen of C5b9 (monoclonal murine 2A1 antibody) as the detecting antibody, and to C6 (polyclonal anti-human C6, crossreactive with rat C6) as the capturing antibody, as described elsewhere (11).

Isolation of glomerular RNA and Northern blotting. To investigate whether the glomerular bFGF synthesis is upregulated in PHN, total glomerular RNA was extracted from isolated glomeruli of normal rats or rats with PHN at day 5 after disease induction using RNAzol B (Cinna/Biotecx Laboratories, Friendswood, TX) followed by a $\mathrm{LiCl}$ precipitation as described previously (16). Northern analysis was performed as described (24) using $30 \mu \mathrm{g}$ of denatured RNA per lane. A

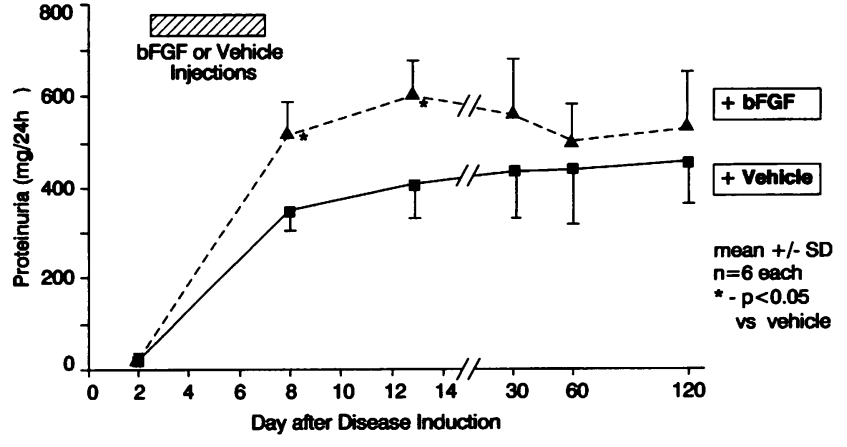

Figure 2. Proteinuria in PHN rats following five i.v. injections of either vehicle or bFGF.

539-bp, radiolabeled (24) XHO1/NCO1 fragment of rat ovarian bFGF cDNA from plasmid RObFGF-477 (kind gift of A. Baird, Whittier Institute, La Jolla, CA) was used to detect rat bFGF transcripts. Membranes were prehybridized and hybridized as described and autoradiograms were obtained (24).

Miscellaneous measurements. Urinary protein was measured using the Bio-Rad Protein Assay (Bio-Rad Laboratories GmbH, München, Germany) and bovine serum albumin (Sigma Chemical Co., St. Louis, MO) as a standard. Creatinine was measured in serum or urine using an autoanalyzer (Beckman Instruments GmbH, München, Germany).

Statistical analysis. All values are expressed as mean \pm SD. Statistical significance (defined as $P<0.05$ ) was evaluated using the Student's $t$ test (25).

\section{Results}

bFGF augments proteinuria and aggravates glomerular injury during early PHN (day 0 to 13). Fig. 2 shows that marked proteinuria developed beyond day 3 after disease induction in vehicle-injected PHN rats. In the PHN rats that had received injections of bFGF, proteinuria was significantly increased $(P$ $<0.05$ ) over the vehicle-injected rats at days 8 and 13 (Fig. 2).

Light microscopy of PAS-stained renal sections from vehicle-injected PHN rats showed mild to moderate podocyte hypertrophy but no other glomerular abnormalities at days 5 and 8 after disease induction (Fig. $3 a$ ). In contrast, in bFGF-injected PHN rats podocytes appeared considerably hypertrophied, associated with extensive pseudocyst formation (Fig. $3 b$ ). Podocyte cell nuclei were frequently very large. The number of glomerular mitoses in bFGF-injected PHN rats increased 8.5-fold on day 5 and 4.5 -fold on day 8 over that observed in vehicle treated rats (Fig. 4). The majority of these mitoses were located at the edge of the glomerular tuft (Figs. $3 c$ and 4), suggesting that they were confined to podocytes. Rare podocytes appeared to be multinucleated (Fig. $3 d$ ). No effect of bFGF on the frequency of mitoses in parietal glomerular epithelial cells or tubulointerstitial cells (data not shown) was noted. Total glomerular nuclei counts increased significantly between days 5 and 8 in bFGF injected PHN rats, but not in vehicle treated PHN rats (Table I). In contrast, the number of apoptotic cells per glomerular cross section remained constant in both groups (Table I).

Electron microscopy of renal biopsies obtained at day 5 and 8 after induction of PHN showed typical foot process "fusion" and subepithelial immune deposits in vehicle-injected PHN rats (Fig. $5 a$ ). In addition to these findings, in bFGF-injected PHN 

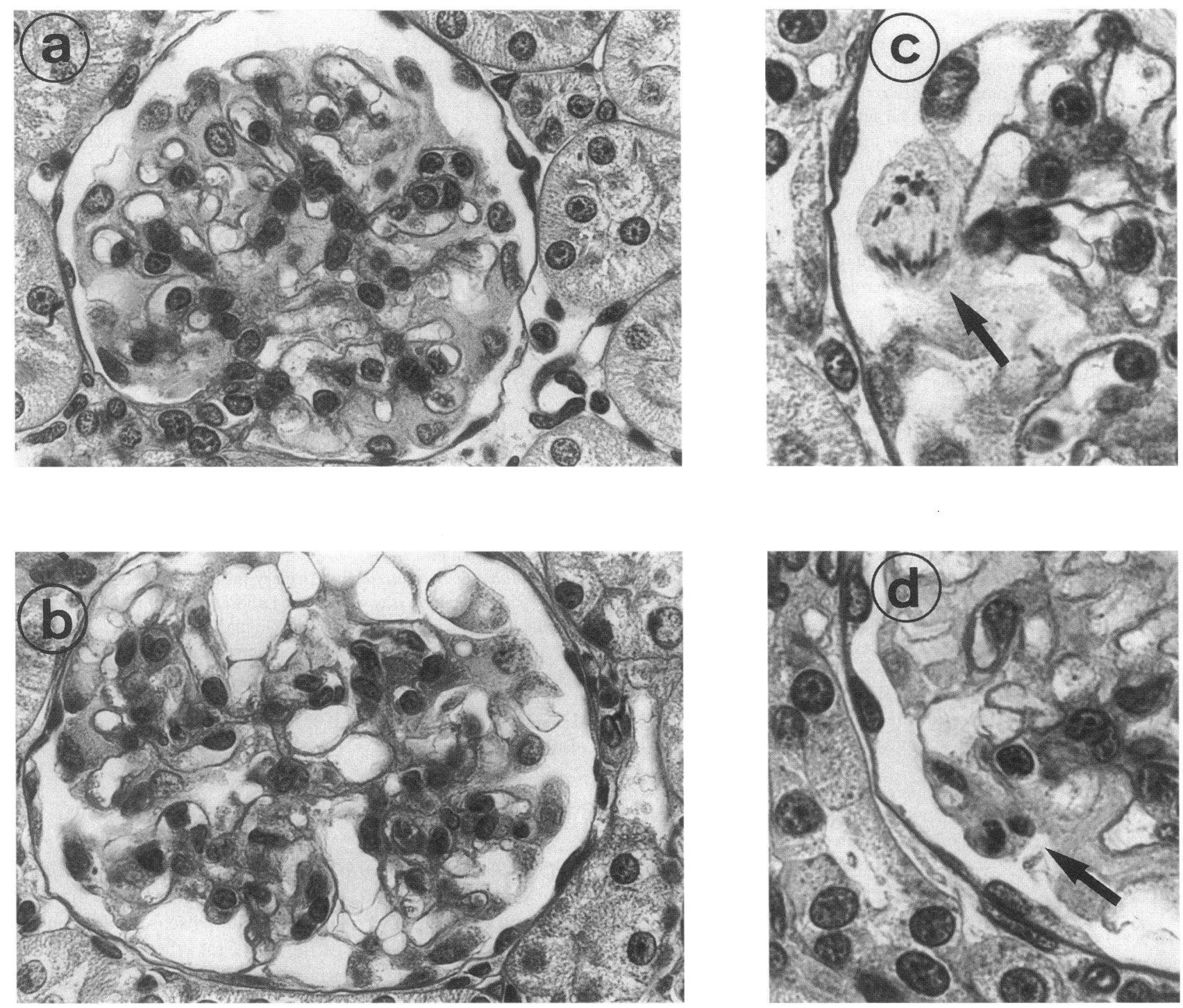

Figure 3. Renal morphology of vehicle-injected ( $a$ ) or bFGF-injected ( $b$ ) PHN rats on day 5 after disease induction. Podocyte hypertrophy but no other abnormalities are noted in vehicle-injected PHN rats, while there is marked podocyte hypertrophy and pseudocyst formation in bFGF-injected PHN rats. Frequent podocyte mitoses were observed in bFGF-injected PHN rats ( $c$, arrow) and, rarely, podocytes which apparently contained more than one nucleus could be detected $(d, a r r o w)$. PAS-stain, magnifications $400(a$ and $b)$ and 1,000 $(c$ and $d)$.

rats, podocytes were characterized by marked hypertrophy and attenuation of the cell bodies, nuclear enlargement, and extensive pseudocyst formation (Fig. $5 b$ ). Protein reabsorption droplets were more frequently observed in podocytes of bFGF-injected PHN rats than in those of vehicle injected PHN rats. Mitotic figures and binucleated podocytes were also seen at the electron microscopy level. Podocyte cell bodies were often densely filled with absorption droplets of varying electron density. Although rare, areas of podocyte detachment from the GBM were clearly seen (Fig. $5 b$ ).

Renal biopsies were also immunostained for desmin, a cytoskeletal marker of podocyte injury (26), as well as $\alpha$-smooth muscle actin, a cytoskeletal marker of activated mesangial cells (24). Similar to previous observations (26), desmin staining within the core of the glomerular tuft and, in particular, along the edge of the glomerular tuft increased in vehicle-injected PHN rats (Fig. 6). Desmin immunostaining of the core and edge of the glomerular tuft increased further in bFGF-injected
PHN rats (Fig. 6). No glomerular expression of $\alpha$-smooth muscle actin was detectable in either group.

The normal immunostaining pattern with anti-bFGF antibody, i.e., strong mesangial staining and weaker podocyte staining (3), was not altered in vehicle-injected PHN rats. Mesangial staining for bFGF tended to be mildly increased in bFGF-injected PHN rats (Table I) but podocyte staining was unchanged. No bFGF mRNA could be detected by Northern blotting in normal rat glomeruli or glomeruli isolated from PHN rats at 5 d after disease induction (data not shown).

Immunostaining for the extracellular matrix proteins laminin and type IV collagen confirmed previous observations (27) by showing a normal glomerular staining pattern in vehicleinjected PHN rats on days 5 and 8. In bFGF-injected PHN rats, podocytes and the glomerular basement membrane continued to exhibit a normal staining pattern, but mild expansion of immunostaining in the mesangium was occasionally noted on days 5 and 8 (Table I). 


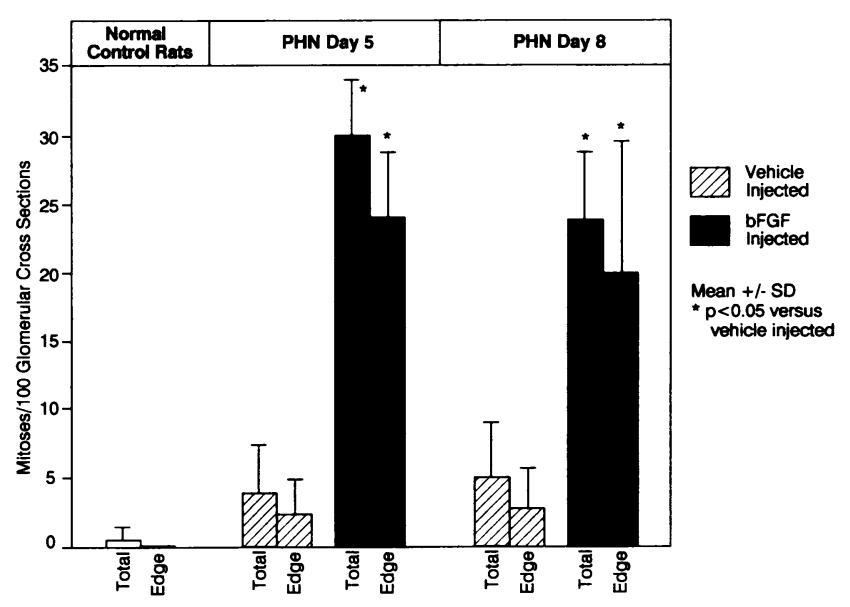

Figure 4. Glomerular mitoses in vehicle or bFGF-injected PHN rats on days 5 and 8 after disease induction. Mitoses (as defined by the number of mitotic figures) are expressed as either the total number of mitoses within the core of the glomerular tuft or mitoses located at the edge of the glomerular tuft. For comparison, values obtained in normal, nonmanipulated rats are shown as well.

No glomerular influx of neutrophils or lymphocytes was observed in vehicle or bFGF-injected PHN rats during the early phase of the disease. The number of glomerular macrophages in vehicle-injected PHN rats increased 1.5-fold (day 5) and 1.3fold (day 8 ) over normal control specimens $(1.1 \pm 0.2$ macrophages/glomerular cross section). This finding remained unchanged in bFGF-injected PHN rats (Table I).

To exclude the possibility that the bFGF injections affected the binding of sheep anti-Fx1A antibody to podocytes, glomerular sheep IgG deposition was quantified on day 5 after the induction of PHN in both vehicle and bFGF-injected rats. The data showed no difference between the two groups (fluorescence intensity in vehicle-injected PHN rats $1.36 \pm 0.45$ fluorescence units versus $1.44 \pm 0.36$ fluorescence units in bFGF-in-

Table I. Glomerular Nuclei, Apoptotic Cells, bFGF, Matrix Protein, and Macrophage Content

\begin{tabular}{|c|c|c|c|c|}
\hline & \multicolumn{2}{|c|}{ PHN day 5} & \multicolumn{2}{|c|}{ PHN day 8} \\
\hline & + Vehicle & $+\mathrm{bFGF}$ & + Vehicle & $+\mathrm{bFGF}$ \\
\hline $\begin{array}{l}\text { Nuclei per glomerular cross } \\
\text { section (mean) }\end{array}$ & $53 \pm 3$ & $50 \pm 1$ & $55 \pm 4$ & $56 \pm 1 *$ \\
\hline $\begin{array}{l}\text { Apoptoses per } 50 \text { glomerular } \\
\text { cross sections }\end{array}$ & $3.0 \pm 1.0$ & $3.8 \pm 2.5$ & $3.5 \pm 1.3$ & $3.0 \pm 1.0$ \\
\hline bFGF (score) & $1.5 \pm 0.3$ & $1.7 \pm 0.2$ & $1.5 \pm 0.2$ & $1.8 \pm 0.3$ \\
\hline Type IV collagen (score) & $1.4 \pm 0.2$ & $1.6 \pm 0.2$ & $1.3 \pm 0.1$ & $1.7 \pm 0.2 \ddagger$ \\
\hline Laminin (score) & $1.6 \pm 0.1$ & $1.8 \pm 0.4$ & $1.5 \pm 0.2$ & $1.7 \pm 0.1$ \\
\hline $\begin{array}{l}\text { Monocytes/macrophages per } \\
\text { glomerular cross section }\end{array}$ & $1.7 \pm 0.5$ & $2.0 \pm 0.5$ & $1.4 \pm 0.4$ & $1.7 \pm 0.6$ \\
\hline
\end{tabular}

Glomerular nucleus counts, apoptotic cells, and immunohistochemical staining scores of the glomerular tuft for bFGF, type IV collagen, and laminin as well as glomerular monocyte/macrophage counts in vehicle or bFGF-injected PHN rats. Means \pm SD; $* P<0.05$ PHN day 5 vs day 8 within the same group; ${ }^{\ddagger} P<0.05$ vehicle vs bFGF-injected PHN rats.
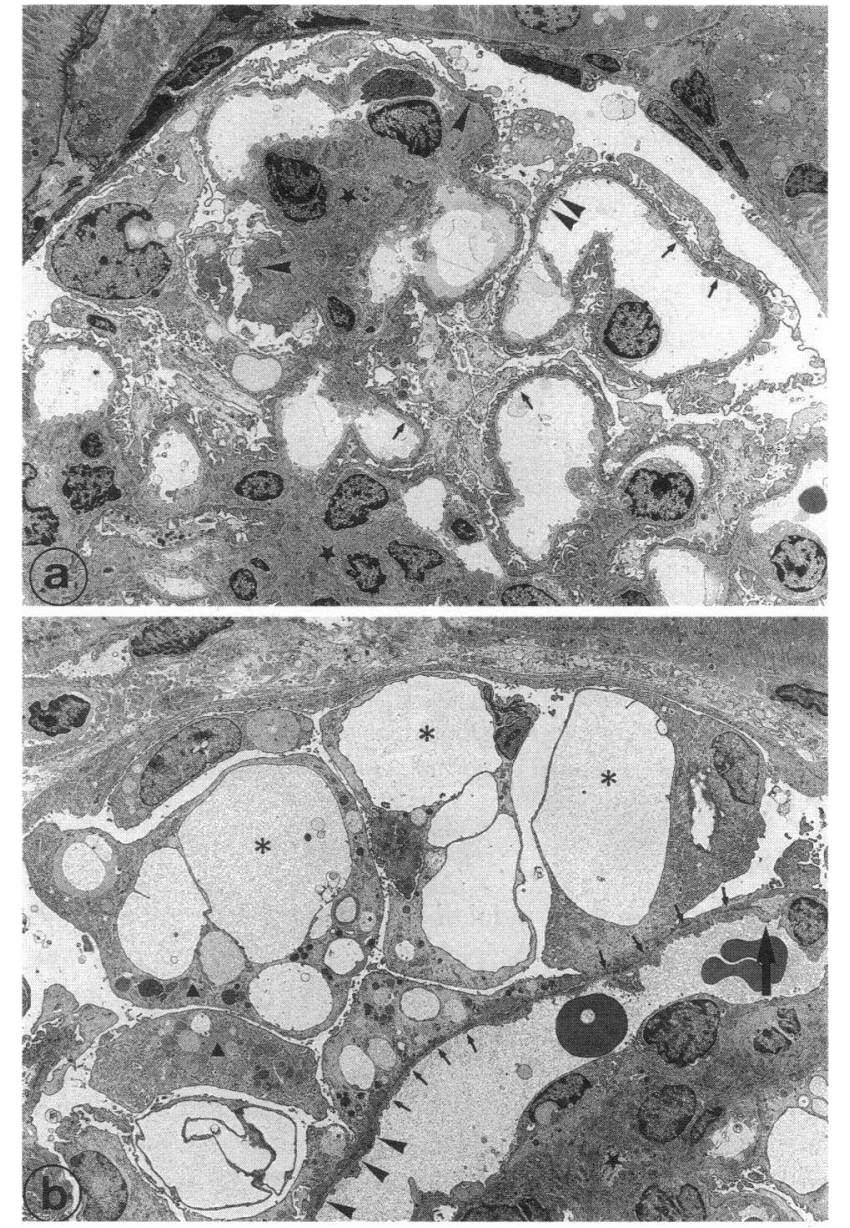

Figure 5. Electron microscopic appearance of vehicle and bFGF-injected PHN rats on day 5 after disease induction. (a) Typical PHN lesions are seen, including subepithelial immune deposits (arrowheads) and mesangial expansion (stars). Podocytes show increased amounts of lysosomal elements and of surface microvilli, but the interdigitation pattern of the foot processes is generally preserved (arrows). (b) In addition to immune deposits (arrowheads) and mesangial expansion (stars), severe podocytic lesions are seen, including cell hypertrophy with extensive pseudocyst formation (asterisks), widespread footprocess "fusion" (arrows), and massive accumulation of absorption droplets in podocyte cell bodies (triangles). Detachment of podocytes from the GBM is seen in one area (thick arrow). Magnification 2,000.

jected PHN rats; $P>0.05$ ). Also, glomerular C3 deposition was not different between the two groups and ranged from $2+$ to $3+$ in all specimens. Finally, the urinary excretion of C5b9, a direct reflection of ongoing podocyte immune injury (11), was not altered by bFGF injections in PHN rats on day 5 ( $1.2 \pm 0.5$ versus $1.3 \pm 0.7 \mathrm{U} / \mathrm{mg}$ creatinine in vehicle and bFGFinjected PHN rats, respectively) and day $8(0.5 \pm 0.3$ versus $0.4 \pm 0.2 \mathrm{U} / \mathrm{mg}$ creatinine). Increased urinary C5b-9 excretion was no longer detectable at days $13,30,60$, and 120 after induction of PHN in either group.

bFGF aggravates the development of glomerulosclerosis in late PHN (day 30 to 120). No glomerulosclerosis was noted at day 30 after disease induction in PHN rats injected with bFGF or vehicle (Table II ) $60 \mathrm{~d}$ after disease induction bFGF-injected PHN rats exhibited a minor increase of segmental glomerulosclerosis over that observed in vehicle-injected PHN rats (Table 


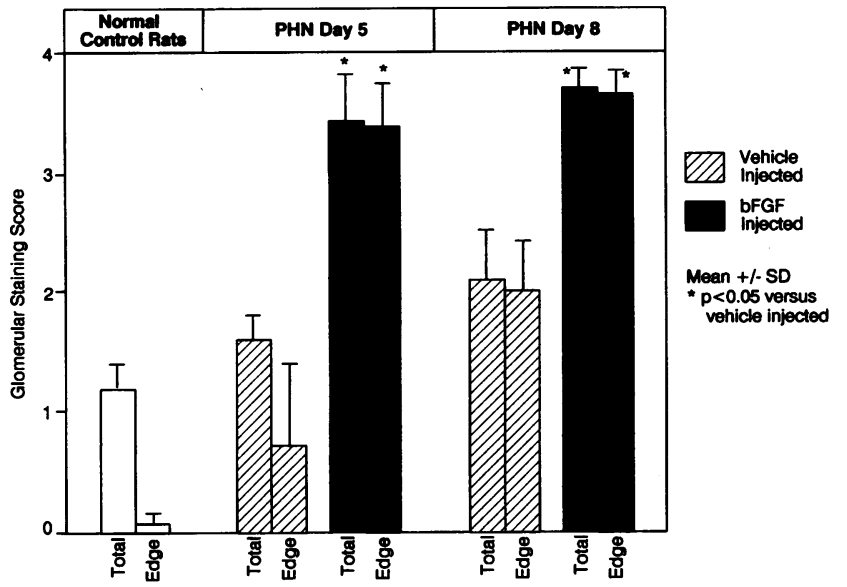

Figure 6. Glomerular expression of desmin in vehicle or bFGF-injected PHN rats on days 5 and 8 after disease induction. Desmin immunostaining scores are expressed as either the score of the total glomerular tuft or the score of the edge of the glomerular tuft (see Methods). For comparison, values obtained in normal, nonmanipulated rats are shown as well.

II). $120 \mathrm{~d}$ after disease induction, a marked difference in the number of segmentally or globally sclerosed glomeruli was noted between bFGF and vehicle-injected PHN rats (Table II, Fig. 7). The development of glomerulosclerosis was associated with periglomerular fibrosis, mild to moderate interstitial fibrosis and interstitial mononuclear cell infiltration, and occasional tubular dilatation (Fig. 7). These changes were more pronounced in the PHN rats that had received bFGF than in those that had received vehicle only (Fig. 7).

In accordance with our observations in other models of progressive glomerular sclerosis (28), the development of glomerulosclerosis in bFGF-injected PHN rats was associated with an increase in silver-staining extracellular matrix as well as diffusely increased immunostaining for glomerular laminin and type IV collagen (data not shown).

Serum creatinine was not different between bFGF- and vehicle-injected PHN rats until day 120 after disease induction (Table II). On day 120 , serum creatinine concentrations were sig-

Table II. Glomerulosclerosis and Renal Function

\begin{tabular}{lrcc}
\hline & $\begin{array}{c}\text { Vehicle- } \\
\text { injected } \\
\text { PHN rats }\end{array}$ & $\begin{array}{c}\text { bFGF- } \\
\text { injected } \\
\text { PHN rats }\end{array}$ \\
\hline Degmental or global glomerulosclerosis* & 30 & $0 \pm 0$ & $0 \pm 1$ \\
$\quad$ (\% of glomerular cross sections) & 60 & $4 \pm 3$ & $8 \pm 5$ \\
& 120 & $13 \pm 5$ & $39 \pm 18^{\ddagger}$ \\
Serum creatinine (mg/dl) & 30 & $0.5 \pm 0.1$ & $0.6 \pm 0.1$ \\
& 60 & $0.6 \pm 0.1$ & $0.6 \pm 0.2$ \\
& 120 & $0.7 \pm 0.1$ & $1.2 \pm 0.2^{\ddagger}$
\end{tabular}

Percentage of glomeruli exhibiting segmental or global sclerotic changes as well as serum creatinine concentrations in PHN rats injected with either vehicle or $\mathrm{bFGF}$ from day 3 to 8 after disease induction.

Mean \pm SD, $n=6$ each. ${ }^{*}$ Less than $25 \%$ of the glomeruli exhibited global glomerulosclerosis. ${ }^{\ddagger} P<0.05$ vehicle vs bFGF-injected PHN rats.
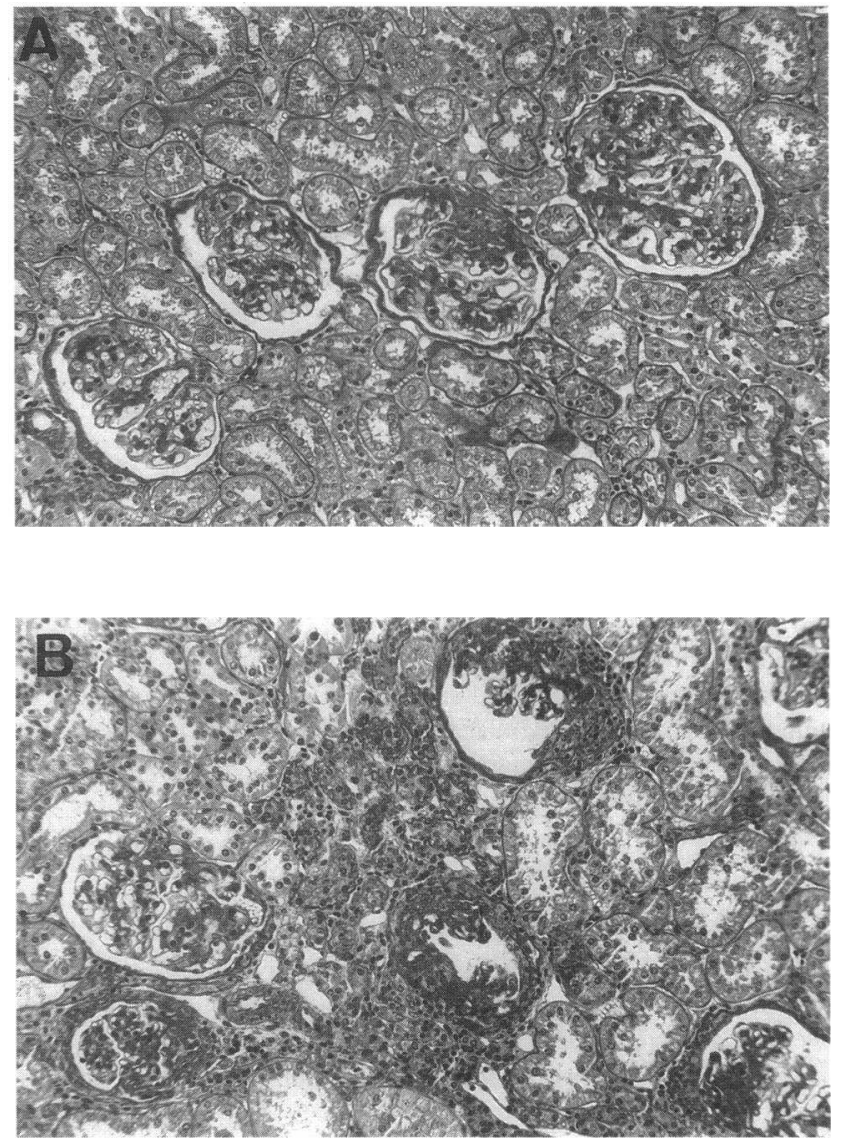

Figure 7. Renal morphology in vehicle- $(A)$ or bFGF-injected $(B)$ PHN rats on day 120 after disease induction. In the vehicle-injected PHN rat, occasional focal glomerulosclerosis is noted, while the remaining renal tissue exhibits a normal morphology. In the bFGF-injected PHN rat, many glomeruli exhibit focal or global glomerulosclerosis, and some degree of interstitial fibrosis as well as infiltration of the interstitium with inflammatory cells is noted. PAS-stain, magnification 100 .

nificantly higher in bFGF-injected PHN rats than in vehicleinjected PHN rats (Table II). As shown in Fig. 2, proteinuria did not differ between the two groups at any of the late time points.

bFGF-induced glomerular injury depends on complement activation during early PHN. To elucidate the mechanism(s) involved in the aggravation of PHN by bFGF several control experiments were carried out. Since the main pathological characteristic of PHN, namely proteinuria, is believed to require the insertion of C5b-9 into the podocyte membrane $(1,2)$, the control experiments focused primarily on the role of complement. Thus, rats were either depleted of complement with CVF before the induction of PHN (PHN/CVF rats), or they were injected with anti-gp 330, an antibody fraction of the antiFx1A antiserum, which does bind to podocytes but does not activate complement (2)(anti-gp 330 rats).

Immunohistological examination of renal tissue obtained from PHN/CVF and anti-gp 330 rats injected with bFGF showed $2+$ to $3+$ glomerular deposition of sheep IgG in all glomeruli but no glomerular $\mathrm{C} 3$ deposits. There was no evidence of podocyte injury in either group as evidenced by $(a)$ the lack of proteinuria, $(b)$ the absence of podocyte mitoses or pseudocysts, and $(c)$ minimal or absent desmin immunostaining 
Table III. Effects of Poly-D-Lysine in PHN Rats

\begin{tabular}{|c|c|c|c|}
\hline & $\begin{array}{l}\text { Day after } \\
\text { disease } \\
\text { induction }\end{array}$ & $\begin{array}{l}\text { PHN + } \\
\text { vehicle }\end{array}$ & $\begin{array}{c}\text { PHN + } \\
\text { poly-D-lysine }\end{array}$ \\
\hline \multirow[t]{4}{*}{ Proteinuria $(\mathrm{mg} / 24 \mathrm{~h})$} & 5 & n.d. & n.d. \\
\hline & 8 & $342 \pm 39$ & $1,993 \pm 1,010^{\| \prime}$ \\
\hline & 30 & $396 \pm 64$ & $482 \pm 93$ \\
\hline & 120 & $418 \pm 70$ & $466 \pm 130$ \\
\hline \multicolumn{4}{|l|}{ Mitoses at the edge of the } \\
\hline \multirow[t]{2}{*}{ glomerular tuft* } & 5 & $3.8 \pm 3.6$ & $0.9 \pm 1.2$ \\
\hline & 8 & $5.0 \pm 3.9$ & $2.4 \pm 2.3$ \\
\hline \multicolumn{4}{|l|}{ Desmin staining: } \\
\hline Tuft $^{\ddagger}$ & 5 & $1.2 \pm 0.1$ & $1.3 \pm 0.3$ \\
\hline Edge $^{\S}$ & 5 & $0.4 \pm 0.2$ & $0.6 \pm 0.5$ \\
\hline Tuft $^{\ddagger}$ & 8 & $2.3 \pm 0.4$ & $2.1 \pm 0.9$ \\
\hline Edge $^{\S}$ & 8 & $2.0 \pm 0.3$ & $1.8 \pm 1.1$ \\
\hline \multicolumn{4}{|l|}{ Segmental or global } \\
\hline glomerulosclerosis (\%) & 120 & $13 \pm 5$ & $19 \pm 5$ \\
\hline Serum creatinine $(\mathrm{mg} / \mathrm{dl})$ & 120 & $0.7 \pm 0.1$ & $0.7 \pm 0.2$ \\
\hline
\end{tabular}

Functional and morphological findings in PHN rats injected with polyD-lysine as compared to vehicle-injected PHN rats. * Expressed as mitoses per 100 glomerular cross sections. ${ }^{\ddagger}$ Mean immunostaining score of the total glomerular tuft. ${ }^{8}$ Mean immunostaining score of the edge of the glomerular tuft. " $P<0.05 \mathrm{PHN}+$ poly-D-lysine vs. PHN + vehicle. n.d., not determined.

in podocytes. However, in both groups, bFGF led to a small increase in mitoses within the core of the glomerular tuft (range $2.1 \pm 0.8$ - to $3.4 \pm 1.2$-fold ) when compared with normal control specimens. In many cases, these mitoses appeared to be localized in glomerular endothelial cells (data not shown).

bFGF-induced glomerular injury exhibits different characteristics than those induced by injections of another cationic compound. To evaluate whether the effects of bFGF in PHN are related to the positive charge of bFGF at physiological $\mathrm{pH}$, control experiments were performed in which $\mathrm{PHN}$ rats received five injections of poly-D-lysine, a strongly cationic polyamino acid. The findings on day 8 showed that poly-D-lysine markedly augmented proteinuria when compared to vehicle-injected PHN rats (Table III). Similar to bFGF injected PHN rats, the increase in proteinuria induced by poly-D-lysine was transient and did not persist until day 120 (Fig. 2 and Table III). PAS staining of renal biopsies revealed moderate podocyte hypertrophy. However, in contrast to bFGF-injected PHN rats podocyte pseudocyst formation was very rare. Furthermore, compared with vehicle-injected PHN rats, the number of podocyte mitoses tended to decrease rather than increase in the poly-D-lysine injected PHN rats (Table III). In contrast to bFGF-injected PHN rats, no further upregulation of podocyte desmin expression was noted in poly-D-lysine injected PHN rats (Table III). In the long term, injections of poly-D-lysine into PHN rats did not significantly alter the frequency of segmental or global glomerulosclerosis (Table III).

The glomerular actions of bFGF depend on the degree of immunological glomerular injury. Given the absence of podocyte injury in PHN/CVF rats and anti-gp 330 rats, and given our previous observations in mesangial cells $(3,4)$, we hypothesized that some degree of podocytic immune injury may be necessary to render these cells suspectible to the action of bFGF.

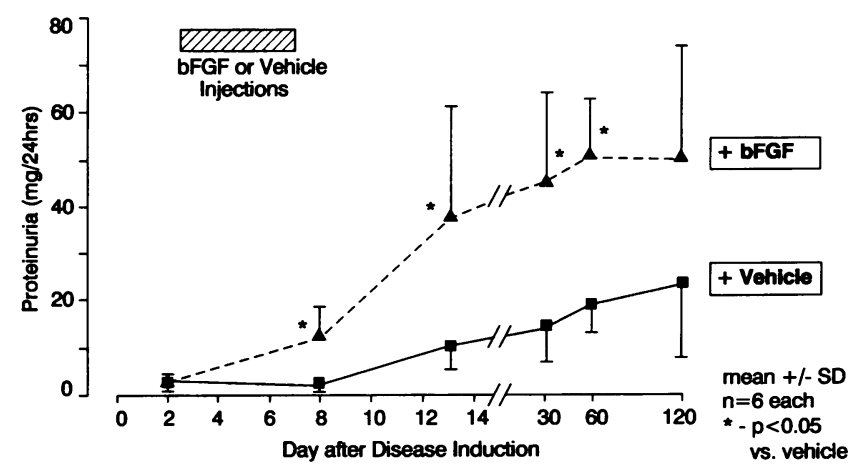

Figure 8. Proteinuria in vehicle- or bFGF-injected rats that had received $10 \%$ of the regular anti-Fx1A dose on day 0.

Therefore, the effects of bFGF were examined in rats injected with 10 or $5 \%$ of the regular anti-Fx1A dose and in normal rats that received PBS only.

In the rats that had received $10 \%$ of the regular anti-Fx $1 \mathrm{~A}$ dose plus vehicle injections from day 3 to 8 , minor proteinuria developed during the autologous phase on day 12 , which persisted until day 120 (Fig. 8). However, no increase in the number of glomerular mitoses (Fig. 9) or in the desmin immunostaining (Fig. 10) over normal controls was noted. If the rats received bFGF instead of vehicle injections, proteinuria was significantly increased on days 13,30 , and 60 (Fig. 8), as did the number of glomerular mitoses at days 5 and 8 (Fig. 9). In this case, apparently, most of the mitoses were due to proliferating endothelial and/or mesangial cells, as few mitoses were noted at the edge of the glomerular tuft (Fig. 9). A minor but significant increase of desmin immunostaining, both within the core and at the edge of the tuft, was also observed in the bFGFinjected group (Fig. 10). The frequency of focal or global glomerulosclerosis on day 120 was not significantly different between the vehicle and bFGF-injected group (vehicle-injected: $2 \pm 2 \%$, bFGF-injected: $4 \pm 2 \%$ ).

In the rats, that had received $5 \%$ of the regular anti-Fx $1 \mathrm{~A}$ dose plus either vehicle or bFGF injections, no proteinuria de-

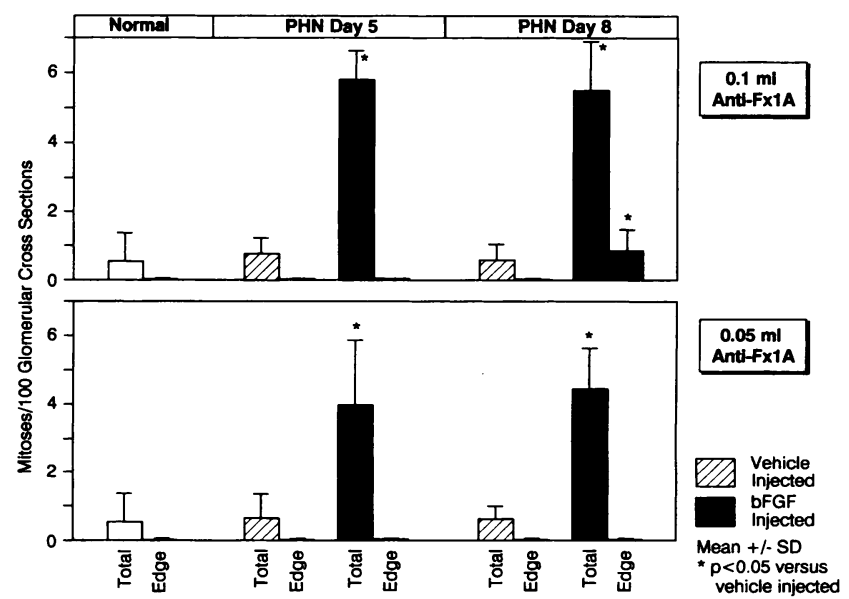

Figure 9. Glomerular mitoses in vehicle- or bFGF-injected rats that had received 10 or $5 \%$ of the regular anti-Fx1A dose, counted on days 5 and 8 after disease induction, expressed as either the total number of mitoses within the core of the glomerular tuft or mitoses located at the edge of the glomerular tuft. For comparison, values obtained in normal, nonmanipulated rats are shown as well. 


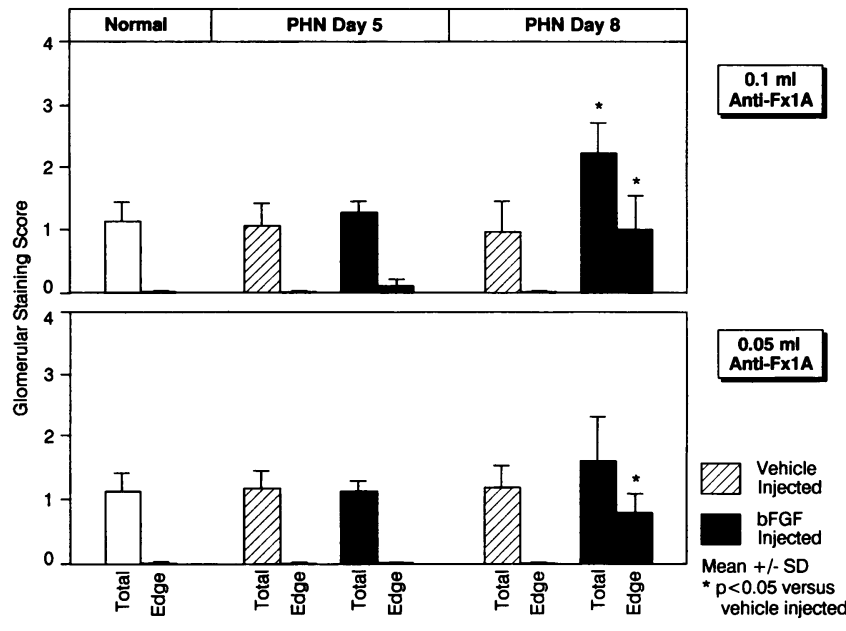

Figure 10. Glomerular expression of desmin in vehicle- or bFGF-injected rats that had received 10 or $5 \%$ of the regular anti-Fx1A dose, evaluated on days 5 and 8 after disease induction. Desmin immunostaining scores are expressed as either the score of the total glomerular tuft or the score of the edge of the glomerular tuft (see Methods). For comparison, values obtained in nonmanipulated rats are shown as well.

veloped. However, in this group, bFGF also induced some glomerular damage, as evidenced by increased mitoses in cells within the core of the glomerular tuft (Fig. 9) and increased podocytic desmin expression (Fig. 10).

In normal rats, the five bFGF injections did not induce shortor long term glomerular damage and neither proteinuria, increased mitoses, podocyte pseudocysts, increased desmin immunostaining, or glomerulosclerosis were noted (data not shown).

Increased bFGF responsiveness in PHN may partially relate to upregulated bFGF receptor expression in podocytes. Immunofluorescent staining of renal sections from normal control rats did not show expression of bFGF receptor in glomeruli but $1+$ to $2+$ immunofluorescence of the luminal aspect of tubular cells (not shown). Injections of bFGF into normal rats did not lead to detectable glomerular receptor expression. In contrast, in vehicle-injected PHN rats on day 5, there was prominent $(3+$ immunofluorescence) bFGF receptor expression in the glomeruli (Fig. 11). The glomerular staining pattern in these rats followed the GBM and was finely granular along the outer aspect of the GBM (Fig. 11). In addition, some immunoreactivity appeared to localize to the cell body of podocytes (Fig. 11). Immunoreactivity was also detected in the basolateral parts of some tubules (Fig. 11). To further define the glomerular localization of bFGF receptor expression, immunoelectron microscopy was performed. Using this technique, receptor expression was demonstrated in the Golgi apparatus and vesicles of podocytes (Fig. 12). Occasional gold particles were also associated with the cell membrane, but the majority of gold particles localized to the subepithelial immune deposits (Fig. 12). Renal bFGF receptor expression was no longer detectable in PHN rats on day 120 after disease induction. The above findings remained unchanged in bFGF-injected PHN rats. In rats that had previously received injections of either anti-gp 330 or $5 \%$ of the normal anti-Fx1A dose, there was also increased glomerular bFGF receptor expression in a pattern similar to that observed in the PHN rats. However, in these rats, glomerular immuno- fluorescence consistently ranged from $1+$ to $2+$ only, and no tubular immunofluorescence was observed.

\section{Discussion}

The main finding of the present study was that bFGF markedly enhanced glomerular injury in rats with established membranous nephropathy. Acutely, bFGF increased both the expression of the cytoskeletal protein desmin in podocytes as well as the number of podocyte mitoses and pseudocysts. It has been shown $(26,29)$ that desmin can serve as a marker of podocyte injury. Recently, we also reported that podocyte injury in PHN is associated with some degree of podocyte proliferation but failed to identify the mitogenic stimulus in those studies (30). The present data as well as recent in vitro (7) and in vivo findings (31) suggest that bFGF may be one of the factors involved. Our data clearly show that bFGF can induce nuclear division in podocytes resulting in increased nuclear counts per glomerulus. Some evidence, however, suggests that nuclear division in podocytes was not regularly followed by complete cell division: (a) Rare multinucleated podocytes were detected in our study, and $(b)$ Kriz et al. (31) recently did not observe an increase in absolute podocyte numbers despite nuclear divisions in podocytes after long term administration of high doses of bFGF to rats (see below). The existence of binucleated podocytes was also convincingly demonstrated in human focal glomerulosclerosis (Nagata, M., Y. Yamaguchi, and K. Ito, manuscript submitted for publication). The bFGF-induced augmentation of podocyte mitoses in our study was associated with severe structural abnormalities of the podocytic cytoplasm, probably compromising the important role of podocytes in maintaining glomerular permselectivity (32). Not surprisingly, therefore, the induction of podocyte mitoses and cytoplasmic changes by bFGF was associated with increased proteinuria in the PHN rats. It appears unlikely that the cationic charge of bFGF was responsible for the above effects, since injections of the cationic molecule poly-D-lysine into PHN rats resulted in a different morphological picture, a different rate of glomerulosclerosis, and since bFGF did not induce proteinuria in normal rats (in contrast to poly-D-lysine, which induces proteinuria in normal rats [33]). This suggests that we have investigated an intrinsic biological activity of the bFGF molecule rather than a nonspecific effect.

With respect to chronic glomerular injury, PHN per se is associated with the development of focal glomerulosclerosis. However, the extent of glomerulosclerosis and renal insufficiency is relatively minor (this study and reference 34 ). Basic FGF administration to PHN rats markedly enhanced the development of chronic renal injury as evidenced by increased glomerulosclerosis, interstitial fibrosis, and serum creatinines. Our study therefore supports the hypothesis that the degree of podocyte injury, leading to early focal adhesions between the GBM and Bowman's capsule and subsequent glomerular matrix accumulation, is an important determinant in the pathogenesis of chronic renal failure (35).

To investigate the mechanism(s) underlying the deleterious action of bFGF in PHN, the role of complement, the central mediator of proteinuria in membranous nephropathy (1), was assessed. Our study shows that antibody binding alone to podocytes without subsequent complement activation is not sufficient to render them susceptible to the injurious effect of bFGF. Vice versa, bFGF did not alter glomerular anti-Fx1A binding or the 

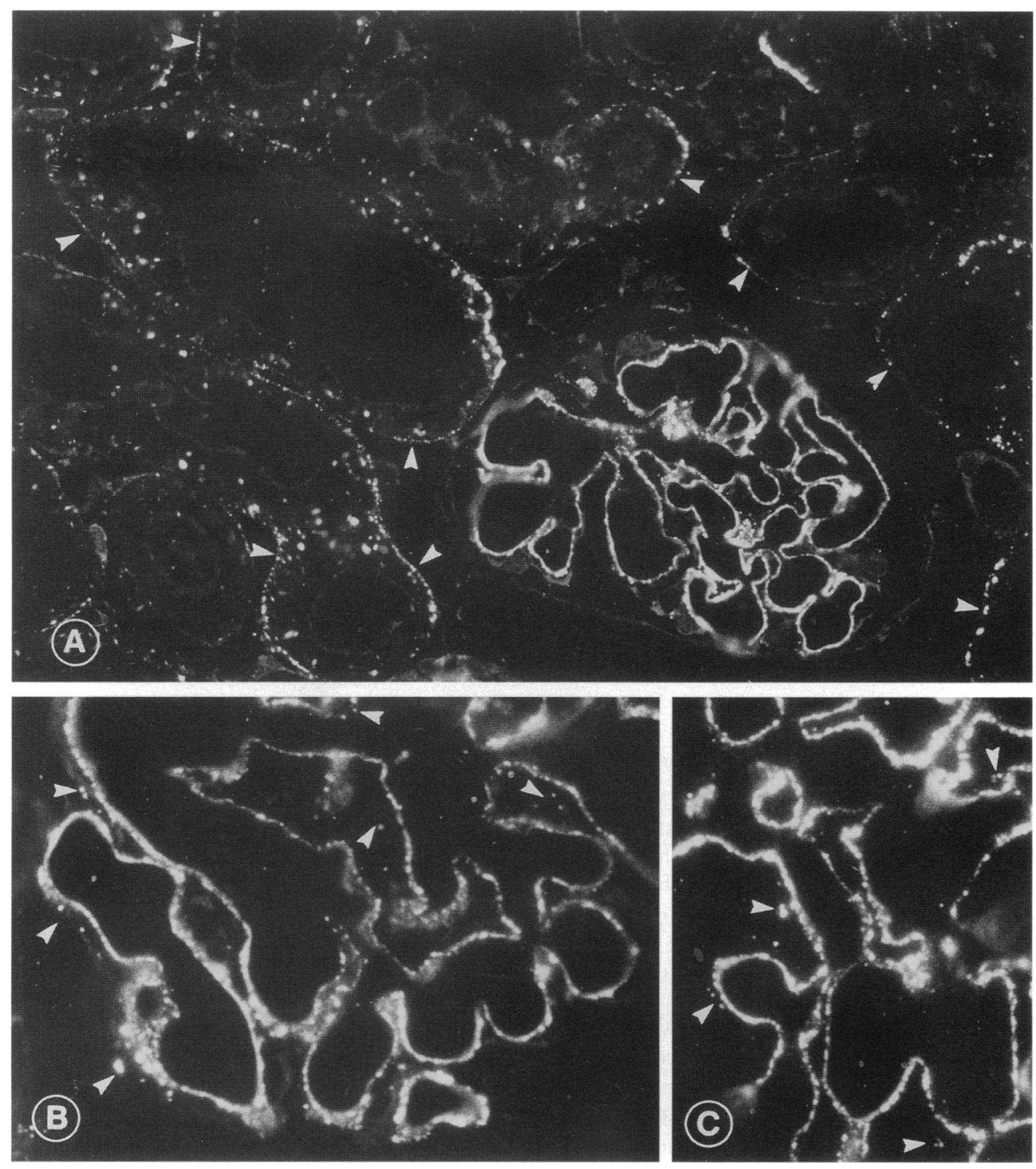

Figure 11. Renal expression of bFGF receptor- $1(\mathrm{flg})$ in PHN rats on day 5 after disease induction. Marked upregulation of glomerular and, to a lesser degree, of tubular (arrows) bFGF receptor-1 expression is present in rats with PHN $(A)$. The finely granular glomerular staining pattern follows the GBM, in particular its outer aspect $(A-$ $C)$. Immunofluorescence is also detected within podocytes ( $B$ and C, arrows).

extent of complement activation in PHN rats as assessed by quantitating antibody deposition using confocal microscopy, immunostaining for complement $\mathrm{C} 3$ and urinary excretion of the membrane attack complex. As discussed above, the charge of bFGF is also unlikely to underlie the enhancement of podocyte injury. Taken together, these observations suggest that prior podocyte injury in some way alters the cellular responsiveness towards bFGF.

In previous studies on mesangioproliferative glomerulonephritis, we had noted that at least a subclinical, complementmediated injury was required to render mesangial cells susceptible to the mitogenic action of bFGF $(3,4)$. Similar observations have been made in vessel walls (36). Also, in the present study bFGF acted as a mitogen for podocytes only if they had suffered some degree of injury before the bFGF infusion. Our data also suggest, that the same is true for endothelial and/or mesangial cells, as some proliferation within the core of the glomerular tuft was noted in bFGF-injected rats that previously had received $10 \%$ of the regular anti-Fx1A doses. This latter observation may relate to the presence of antibodies with antiendothelial cell activity in the anti-Fx1A antiserum (37).
A prime mechanism underlying the increased cellular susceptibility towards the action of bFGF would be modulation of glomerular bFGF receptor expression. Similar to normal human kidney, in which no glomerular bFGF receptor (FGFR-1; $f g$ ) expression has been detected by immunohistochemistry (38), the present study also failed to detect glomerular expression of bFGF receptor in rat glomeruli. In agreement with our findings, a recent in situ hybridization study also failed to detect FGFR1 to FGFR-3 mRNA in most normal rat glomeruli (39). The marked upregulation of bFGFR-1 expression in glomeruli of PHN rats may thus explain why these rats, but not normal rats, showed a marked biological response to bFGF injections. Our data suggest that in PHN the FGFR-1 receptor is synthesized by podocytes, as evidenced by the presence of immunoreactivity in the Golgi apparatus and vesicles of these cells. The preferential localization of FGFR-1 immunoreactivity in subepithelial immune deposits in addition to some immunoreactivity on the surface of podocytes may relate to capping and shedding of the receptor during PHN, a phenomenon which is well established for other podocyte surface molecules such as gp $330(40,41)$. However, some degree of glomerular bFGF receptor upregula- 

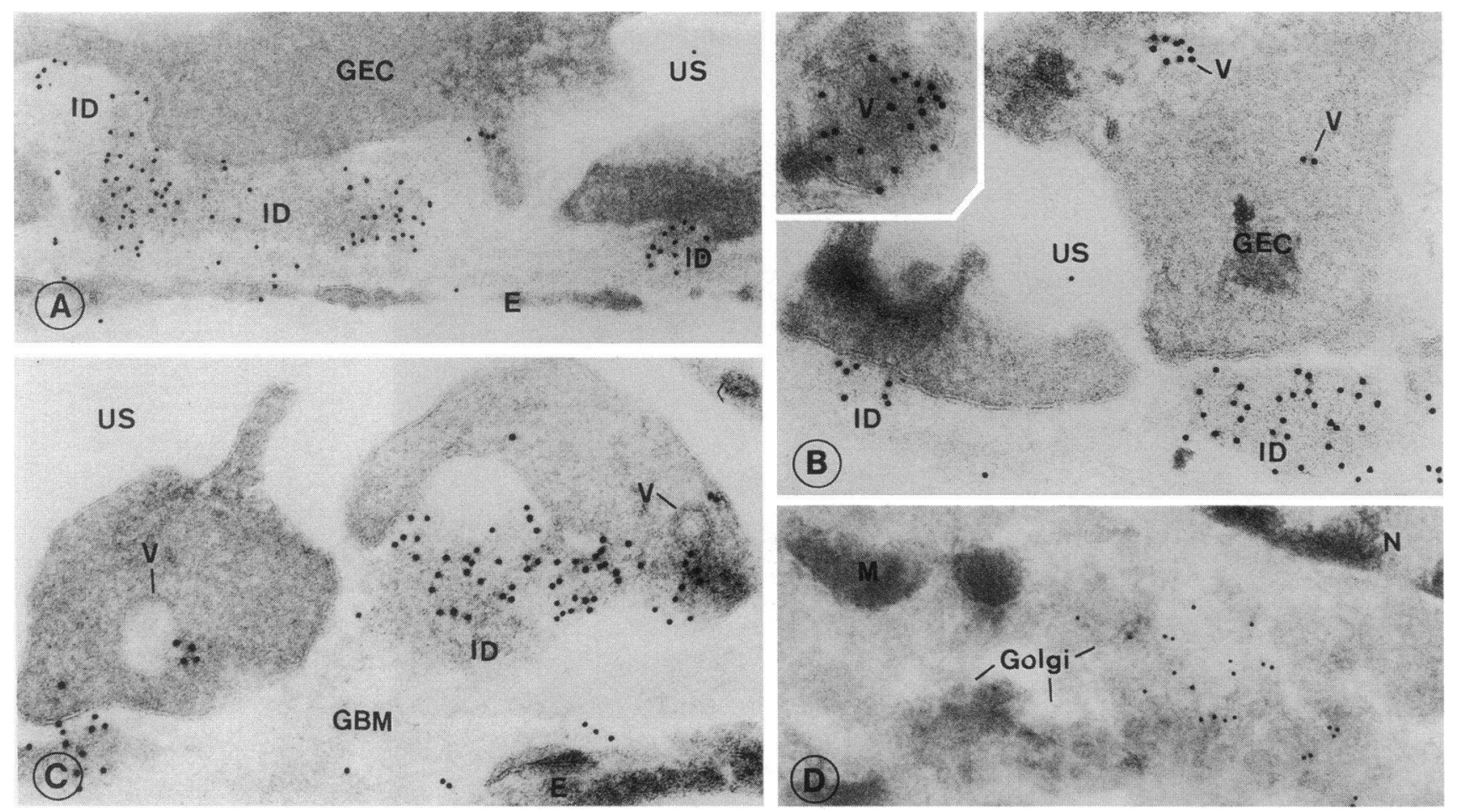

Figure 12. Immunoelectron microscopical expression of bFGF receptor-1 $(\mathrm{flg})$ in glomeruli of PHN rats on day 5 after disease induction. $(A)$ Gold particles localize to subepithelial immune deposits and occasionally to the podocyte cell membrane. $(B)$ Within podocytes, immunoreactivity is present in vesicles. $(C)$ Fusion of bFGF receptor containing vesicles with the cell membrane can occasionally be detected. $(D)$ Immunoreactivity is also present in the Golgi apparatus of podocytes. ID, immune deposits; GEC, glomerular epithelial cell (podocyte); E, endothelial cell; US, urinary space; $\mathrm{V}$, vesicle; $\mathrm{M}$, mitochondrium; $\mathrm{N}$, nucleus.

tion was also noted in anti-gp 330 injected rats not responding to bFGF. This suggests that, apart from bFGF receptor upregulation, additional mechanisms may be involved in the increased bFGF responsiveness in PHN rats. Such mechanisms may include upregulation of FGFR-2 or FGFR-3, postreceptor signalling events or alterations of extracellular heparan sulfate proteoglycan composition, which are of crucial importance for bFGF signalling (42). However, this latter possibility is not supported by our previous studies, which failed to reveal changes in glomerular heparan sulfate proteoglycan staining in early PHN (27).

Two recent studies showed that prior podocyte injury is not an invariable prerequisite for the injurious action of bFGF ( 31 , 43). Thus, a pattern of damage similar to that observed in the present study could be induced by the long term administration of large quantities of bFGF to normal rats: Kriz et al. (31) have shown that the chronic administration (up to $90 \mathrm{~d}$ ) of large quantities of bFGF ( 10-fold higher than the dose used in this study) to normal rats results in proteinuria, podocyte pseudocyst formation, binucleated podocytes, GBM denudation, and focal segmental glomerulosclerosis. Similar findings were also obtained in a study by Mazue et al. (43), who gave normal rats 28 daily i.v. injections of a 20 -fold higher bFGF amount than that used in this study.

In conclusion, this study shows that bFGF can markedly accelerate both acute and chronic podocyte injury with resulting long term damage to the kidney. However, podocytes not only respond to but also synthesize and store bFGF in vivo (7). Based on these observations, we propose the following pathoge- netic mechanism: similar to findings in endothelial cells (44), complement activation and generation of $\mathrm{C} 5 \mathrm{~b}-9$ on the podocyte surface may result in release of preformed podocyte bFGF. Basic FGF released from such injured podocytes may, in turn, stimulate adjacent podocytes, particularly if these adjacent podocytes have been rendered more susceptible to bFGF by prior injury. This mechanism would thus serve to potentiate podocyte damage and consequently glomerulosclerosis. In this context, it is interesting to note that the pathogenesis of HIV-nephropathy, characterized by a rapidly progressive form of focal glomerulosclerosis (45), has recently been linked to bFGF (46). It is also noteworthy that the characteristic podocyte lesions observed in HIV nephropathy, namely enlargement and pseudocyst formation (45), as well as in another, potentially virus-induced disease of the podocyte, namely collapsing glomerulopathy (47), are reminiscent of those observed in the present study. Thus, bFGF-induced podocyte damage may represent a common pathogenetic mechanism by which various forms of glomerular diseases, such as membranous nephropathy and focal glomerulosclerosis, are accelerated.

\section{Acknowledgments}

The authors gratefully acknowledge the generous supply of bFGF by Terri Fields (Synergen), the help of Monika Kregeler and Astrid Fitter in performing the experiments, of Brunhilde Hähnel in performing the electron microscopy studies, and the assistance of Katherine Gordon and Maureen Reilly in conducting the confocal microscopy measurements is also gratefully acknowledged. 
This study was supported in part by the Deutsche Forschungsgemeinschaft (SFB 244/C12), Fonds zur Förderung der Wissenschaft (SFB "Tissue Damage and Repair"), and research grants from the US Public Health Service (DK 34198, DK 47659).

\section{References}

1. Couser, W. G., and C. K. Abrass. 1988. Pathogenesis of membranous nephropathy. Annu. Rev. Med. 39:517-530.

2. Kerjaschki, D. 1990. The pathogenesis of membranous glomerulonephritis: from morphology to molecules. Virchows Arch. B Cell Pathol. 58:253-271.

3. Floege, J., E. Eng, V. Lindner, B. A. Young, M. A. Reidy, and R. J. Johnson. 1992. Rat glomerular mesangial cells synthesize basic FGF: release, upregulated synthesis, and mitogenicity in mesangial proliferative glomerulonephritis. J. Clin. Invest. 90:2362-2369.

4. Floege, J., E. Eng, B. A. Young, and R. J. Johnson. 1993. Factors involved in the regulation of glomerular mesangial cell proliferation in vitro and in vivo. Kidney Int. 43:S47-S54.

5. Klagsbrunn, M., and E. R. Edelman. 1989. Biological and biochemical properties of fibroblast growth factors. Implications for the pathogenesis of atherosclerosis. Arteriosclerosis. 9:269-278.

6. D'Amore, P. A. 1990. Modes for FGF release in vivo and in vitro. Cancer Metastasis Rev. 9:227-238.

7. Takeuchi, A., N. Yoshizawa, M. Yamamoto, Y. Sawasaki, T. Oda, A. Senoo, H. Niwa, and Y. Fuse. 1992. Basic fibroblast growth factor promotes proliferation of rat glomerular visceral epithelial cells in vitro. Am. J. Pathol. 141:107-116.

8. Silver, B. J., F. E. Jaffer, and H. E. Abboud. 1988. Platelet-derived growth factor synthesis in mesangial cells: induction by multiple peptide mitogens. Proc. Natl. Acad. Sci. USA. 86:1056-1060.

9. Alousi, M. A., R. S. Post, and W. Heymann. 1969. Experimental autoimmune nephrosis in rats. Am. J. Pathol. 54:47-71.

10. Salant, D. J., C. Darby, and W. G. Couser. 1980. Experimental membranous glomerulonephritis in rats. Quantitative studies of glomerular immune deposit formation in isolated glomeruli and whole animals. J. Clin. Invest. 66:7181.

11. Schulze, M., P. J. Baker, D. T. Perkinson, R. J. Johnson, R. F. Ochi, R. A. K. Stahl, and W. G. Couser. 1989. Urinary excretion of C5b-9 distinguishes passive Heymann nephritis from other forms of experimental glomerulonephritis in the rat. Kidney Int. 35:60-68.

12. Johnson, R. J., P. Pritzl, H. Iida, and C. E. Alpers. 1991. Platelet-complement interactions in mesangial proliferative nephritis in the rat. Am. J. Pathol. 138:313-321.

13. Susani, M., M. Schulze, M. Exner, and D. Kerjaschki. 1994. Antibodies to glycolipids activate complement and promote proteinuria in passive Heymann nephritis. Am. J. Pathol. 144:807-819.

14. Johnson, R. J., R. L. Garcia, P. Pritzl, and C. E. Alpers. 1990. Platelets mediate glomerular cell proliferation in immune complex nephritis induced by anti-mesangial cell antibodies in the rat. Am. J. Pathol. 136:369-374.

15. Baker, A. J., A. Mooney, J. Hughes, D. Lombardi, R. J. Johnson, and J. Savill. 1994. Mesangial cell apoptosis: the major mechanism for resolution of glomerular hypercellularity in experimental mesangial proliferative nephritis. $J$. Clin. Invest. 94:2105-2116.

16. Floege, J., M. W. Burns, C. E. Alpers, A. Yoshimura, P. Pritzl, K Gordon, R. A. Seifert, D. F. Bowen-Pope, W. G. Couser, and R. J. Johnson. 1992. Glomerular cell proliferation and PDGF expression precede glomerulosclerosis in the remnant kidney model. Kidney Int. 41:297-309.

17. Dijkstra, C. D., E. A. Dopp, P. Joling, and G. Kraal. 1985. The heterogeneity of mononuclear phagocytes in lymphoid organs: distinct macrophage populations in the rat recognized by monoclonal antibodies ED1, ED2, and ED3. Immunology. 54:589-599.

18. Sekiya, S., S. Gotoh, T. Yamashita, T. Watanabe, S. Saitoh, and F. Sendo 1989. Selective depletion of rat neutrophils by in vivo administration of a monoclonal antibody. J. Leukocyte Biol. 46:96-102.

19. Reilly, T. M., D. S. Taylor, W. F. Herblin, M. J. Thoolen, A. T. Chiu, D. W. Watson, and P. B. M. W. M. Timmermans. 1989. Monoclonal antibodies directed against basic fibroblast growth factor which inhibit its biological activity in vitro and in vivo. Biochem. Biophys. Res. Commun. 2:736-743.

20. Floege, J., R. J. Johnson, K. Gordon, H. Iida, P. Pritzl, A. Yoshimura, C. Campbell, C. E. Alpers, and W. G. Couser. 1991. Increased synthesis of extracellular matrix in mesangial proliferative nephritis. Kidney Int. 40:477-488.

21. Adler, S., L. J. Striker, G. E. Striker, D. T. Perkinson, J. Hibbert, and W. G. Couser. 1986. Studies of progressive glomerular sclerosis in the rat. Am. J. Pathol. 123:553-562.

22. Weise, B., T. Janet, and C. Grothe. 1993. Localization of bFGF and FGFreceptor in the developing nervous system of the embryonic and newborn rat. $J$. Neurosci. Res. 34:442-453.

23. Kerjaschki, D., M. Schulze, S. Binder, R. Kain, P. P. Ojha, M. Susani, R.
Horvat, P. J. Baker, and W. G. Couser. 1989. Transcellular transport and membrane insertion of the C 5 b- 9 membrane attack complex of complement by glomerular epithelial cells in experimental membranous nephropathy. J. Immunol. 143:546-552.

24. Johnson, R. J., H. Iida, C. E. Alpers, M. W. Majesky, S. M. Schwartz, P. Pritzl, K. Gordon, and A. M. Gown. 1991. Expression of smooth muscle cell phenotype by rat mesangial cells in immune complex nephritis. J. Clin. Invest. 87:847-858.

25. Wallenstein, S., C. L. Zucker, and J. L. Fleiss. 1980. Some statistical methods useful in circulation research. Circ. Res. 47:1-9.

26. Floege, J., C. E. Alpers, E. H. Sage, P. Pritzl, K. Gordon, R. J. Johnson, and W. G. Couser. 1992. Markers of complement dependent and complement independent glomerular visceral epithelial cell injury in vivo. Lab. Invest. 67:486497.

27. Floege, J., R. J. Johnson, K. Gordon, A. Yoshimura, C. Campbell, L. Iruela-Arispe, C. E. Alpers, and W. G. Couser. 1992. Altered glomerular extracellular matrix synthesis in experimental membranous nephropathy. Kidney Int. 42:573-585.

28. Floege, J., C. E. Alpers, M. W. Burns, P. Pritzl, K. Gordon, W. G. Couser, and R. J. Johnson. 1992. Glomerular cells, extracellular matrix accumulation, and the development of glomerulosclerosis in the remnant kidney model. Lab Invest. 66:485-497.

29. Yaoita, E., K. Kawasaki, T. Yamamoto, and I. Kihara. 1990. Variable expression of desmin in rat glomerular epithelial cells. Am. J. Pathol. 136:899908.

30. Floege, J., R. J. Johnson, C. E. Alpers, C. A. Richardson, K. Gordon, and W. G. Couser. 1993. Visceral glomerular epithelial cells can proliferate in vivo and synthesize PDGF B-chain. Am. J. Pathol. 142:637-650.

31. Kriz, W., B. Hähnel, S. Rösener, and M. Elger. 1995. Long term treatment of rats with FGF-2 results in focal segmental glomerulosclerosis. Kidney Int. In press.

32. Kanwar, Y. S., Z. Z. Liu, N. Kashihara, and E. I. Wallner. 1991. Current status of the structure and functional basis of glomerular filtration and proteinuria. Semin. Nephrol. 11:390-413.

33. Firth, J. D. 1990. Effect of polycations on the function of the isolated perfused rat kidney. Clin. Sci. 79:591-598.

34. Zoja, C., D. Corna, T. Bertani, and G. Remuzzi. 1992. Rats with passive Heymann nephritis and long-lasting proteinuria develop focal segmental glomerulosclerosis. J. Am. Soc. Nephrol. 3:624a. (Abstr.)

35. Kriz, W., M. Elger, M. Nagata, M. Kretzler, S. Uiker, I. Koeppen-Hagemann, S. Tenschert, and K. V. Lemley. 1994. The role of podocytes in the development of glomerular sclerosis. Kidney Int. 45:S64-S72.

36. Lindner, V., R. A. Majack, and M. A. Reidy. 1991. Basic fibroblast growth factor mediates endothelial regrowth and proliferation in denuded arteries. J. Clin. Invest. 85:2004-2008.

37. Jeraj, K., R. L. Vernier, S. P. Sisson, and A. F. Michael. 1984. A new glomerular antigen in passive Heymann's nephritis. Br. J. Exp. Pathol. 65:485498.

38. Hughes, S. E., and P. A. Hall. 1993. Immunolocalization of fibroblast growth factor receptor-1 and its ligands in human tissues. Lab. Invest. 69:173182.

39. Jyo, Y., T. Sasaki, H. Tamai, T. Nohno, N. Itoh, and G. Osawa. 1994 Demonstration of fibroblast growth factor receptor mRNA in glomeruli in mesangial proliferative nephritis by in situ hybridization. J. Am. Soc. Nephrol. 5:784a (Abstr.)

40. Camussi, G., D. Kerjaschki, M. Gonda, T. Nevins, J. C. Rielee, J. Brentjens, and G. Andres. 1989. Expression and modulation of surface antigens in cultured rat glomerular visceral epithelial cells. J. Histochem. Cytochem 37:1675-1687.

41. Kerjaschki, D., and M. G. Farquhar. 1983. Immunocytochemical localization of the Heymann nephritis antigen (gp 330) in glomerular epithelial cells of normal Lewis rats. J. Exp. Med. 157:667-686.

42. Rapraeger, A. C., A. Krufka, and B. B. Olwin. 1991. Requirement of heparan sulphate for bFGF-mediated fibroblast growth and myoblast differentiation. Science (Wash. DC). 252:1705-1708.

43. Mazue, G., F. Bertolero, L. Garofano, M. Brughera, and P. Carminati 1992. Experience with the preclinical assessment of basic fibroblast growth factor. Toxicol. Lett. (Amst.). 64-65 Spec No: 329-328.

44. Benzaquen, L. R., A. Nicholson-Weller, and J. A. Halperin. 1994. Terminal complement proteins C5b-9 release basic fibroblast growth factor and plateletderived growth factor from endothelial cells. J. Exp. Med. 179:985-992.

45. Rao, T. K. S. 1991. Human immunodeficiency virus (HIV) associated nephropathy. Annu. Rev. Med. 42:391-401.

46. Ray, P. E., L. A. Bruggeman, B. S. Weeks, J. B. Kopp, J. L. Bryant, J. W Owens, A. L. Notkins, and P. E. Klotman. 1994. bFGF and its low affinity receptors in the pathogenesis of HIV-associated nephropathy in transgenic mice. Kidney Int. 46:759-772.

47. Detwiler, R. K., R. J. Falk, S. L. Hogan, and J. C. Jennette. 1994. Collapsing glomerulopathy: a clinically and pathologically distinct variant of focal segmental glomerulosclerosis. Kidney Int. 45:1416-1424. 